\title{
An unusual case of mediastinal malignant melanoma with a cardiac metastasis
}

Lakmali MGN, Mudduwa L, Fernando LBM, Mahinda HA

Teaching Hospital, Karapitiya

*Corresponding author: Tel: 0094-718208938. E-mail address: nisansalalakmalia@yaho.com

MLJSL. Vol2. No1. March. pp 22-25

\begin{abstract}
:
Introduction

Mediastinal tumours are often seen in patients aged 30-50 years. Most of them are neurogenic tumours and thymomas. Others include lymphomas, phaeochromocytomas, melanomas, germ cell tumours and thyroid and parathyroid lesions. Although primary cardiac tumours are rare, metastasis in the heart is commoner than primary cardiac tumours.

Case report

A 41 year old woman was admitted to the Teaching Hospital, Karapitiya with a recent onset progressive dyspnoea. She was found to have a rapidly filling pericardial effusion. She died on the third day of admission. A whitish mass infiltrating the full thickness of the right atrial myocardium was found at the autopsy. A similar tumour was present in the anterior mediastinum measuring $7 \times 3 \mathrm{~cm}$. There was no direct connection between the two tumours. H\&E stained sections revealed a similar microscopic appearance in both mediastinal and cardiac masses. The differential diagnosis included lymphoma, melanoma, carcinoma and germ cell tumors. Immunohistochemical staining for LCA, pan cytokeratin, S100 and PLAP were done and only S100 was positive.

\section{Conclusion}

Negative staining for LCA, pan cytokeratin and PLAP excluded the possibility of lymphoma, carcinoma and germ cell tumours. Positive staining for S100 confirmed the diagnosis of melanoma. This was diagnosed as a rare case of primary mediastinal malignant melanoma with a right atrial metastasis causing pericardial effusion.
\end{abstract}

Key words: mediastinal tumours, melanoma, metastasis, heart

\section{Introduction:}

Malignancies are found rarely in the mediastinum. Only $3 \%$ of mediastinal tumours are primary tumours [1]. Out of them $25-49 \%$ are malignant. Primary mediastinal malignancies comprise 55\% lymphomas, $16 \%$ germ cell tumours, $14 \%$ thymomas, 5\% sarcomas, 3\% neurogenic tumours and $7 \%$ other rare tumours [2]. Malignant melanoma belongs to this rare 
survival of malignant melanoma of the skin is extremely poor; one will therefore not expect that of the mediastinum to be any better [4].

\section{Case report:}

A 41 years old mother of 3 children presented to a medical ward complaining of loss of appetite for 2 months, dyspnoea on mild exertion and chest pain for 2 weeks and on and off fever for 1 week. She was found to be having anaemia and features of pericardial and bilateral pleural effusions. Urgent echocardiogram revealed an early cardiac tamponade. Straw coloured pericardial fluid measuring $850 \mathrm{ml}$ was aspirated. It was rapidly filling and $500 \mathrm{cc}$ of fluid was removed during a second pericardiocentesis done on the next day. Microscopic examination of the pericardial fluid was negative for acid fast bacilli but contained malignant cells. Ultrasound scan of the abdomen revealed mild hepatomegaly with a small amount of free fluid in the peritoneum. The patient expired away on the third day of admission while waiting for further investigations.

At autopsy the pericardium contained $100 \mathrm{cc}$ of straw coloured fluid. Heart was enlarged and measured $370 \mathrm{~g}$. There was a mass involving the full thickness of the anterior wall of the right atrium and measured $7 \times 7 \times 1.5 \mathrm{~cm}$ in size (Figure 01). The cut surface appeared whitish with necrotic areas. Bleeding into the myocardium was found. Coronary arteries and the large blood vessels appeared normal.

Figure 01: Cardiac tumour

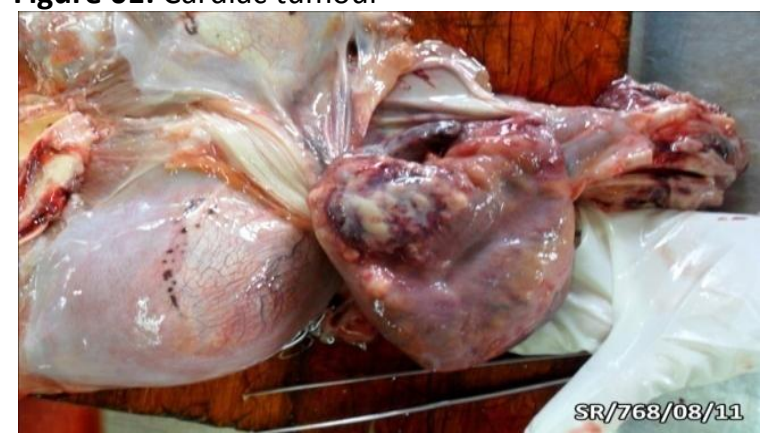

Another whitish mass measuring $7 \times 3 \times 2 \mathrm{~cm}$ in size was found in the middle mediastinum. The tumour was lying anterior to the trachea, above the bifurcation and it encircled the left carotid vessels (Figure 02). The cut surface had a similar appearance with the cardiac tumour. Mediastinal lymph nodes were not enlarged. Mild pulmonary oedema was present. Peritoneum contained $300 \mathrm{cc}$ of ascetic fluid. Liver was congested with no macroscopic metastatic deposits.

Other organs were normal except for the kidneys which showed surface scarring. Sections were obtained from both tumours and each organ for histological examination. Haematoxylin and Eosin stained sections were evaluated (Figure 03).

Figure 2: Mediastinal tumour

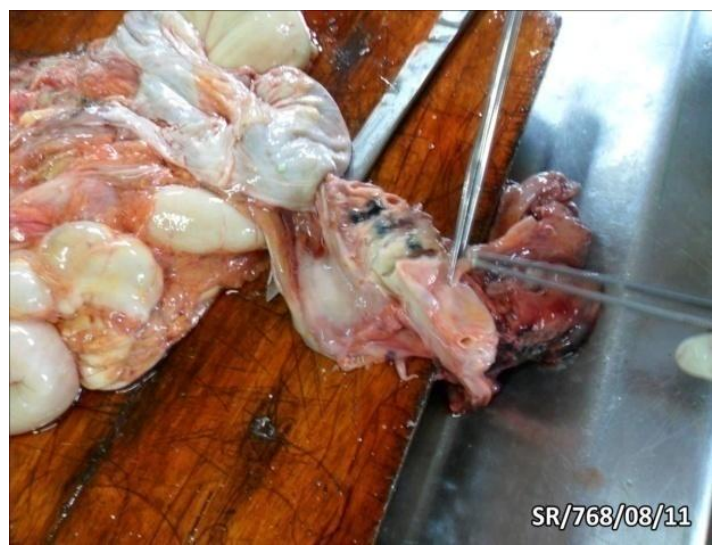

Figure 3: Tumour cells infiltrating the myocardium (H\&E x10)

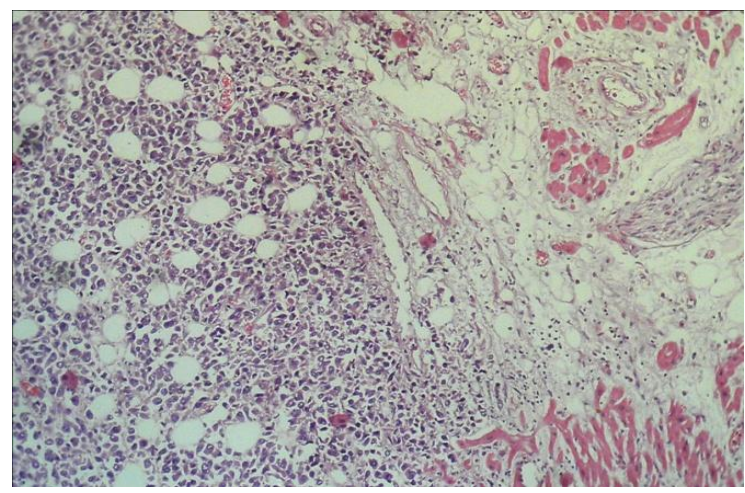


The differential diagnosis included lymphoma, melanoma, epithelial cell tumours and germ cell tumours. Immunohistochemical staining for LCA, pancytokeratin, S100 and PLAP were done and only S100 was positive which was confirmatory of melanoma

\section{Discussion:}

Skin is the most common site for primary malignant melanoma; the most fatal cutaneous neoplasm [5]. Malignant melanomas are developed by neoplastic transformation of melanocytes in the epidermis invading the dermis secondarily. Patients with cutaneous melanomas often develop metastasis at other sites of the skin, lymph nodes and lungs. More than $90 \%$ of malignant melanomas are cutaneous in origin [5]. In addition, melanoma can arise from mucous membranes of nasal cavity, sinuses, lips, buccal mucosa, palate, base of the tongue and tonsils [4]. They can arise in the oesophagus, larynx, gallbladder and leptomeninges rarely. Primary malignant melanoma in the mediastinum is extremely rare, with only a few cases reported to date [6].

A primary malignant melanoma of the mediastinum could be confirmed on the following grounds $[4,5,7]$.

I. No history suggestive of previous melanoma on skin.

II. No demonstrable melanoma was found in the skin or other organs during the autopsy.

III. Tumour morphology compatible with that of a melanoma.

IV. Confirmation by immunohistochemical staining for S100.

There are several explanations as to how these primary melanomas developed in the mediastinum. The most likely explanation is that these tumours arise from melanocytes which have migrated along with the down growth of the primordial respiratory tract during the intrauterine life from the primitive foregut [7]. Ectopic naevus cells in the mediastinal lymph nodes or thymus may undergo malignant change and produce primary melanoma of the anterior mediastinum [7]. Alternatively tumours may arise from areas of squamous metaplasia in which some of the epithelial cells have undergone differentiation into melanocytes [7].

Almost half of mediastinal tumours cause no symptoms and are found on a chest $x$ ray done for some other reason. Symptoms that do occur are due to compression of local structures and may include: chest pain, chills, cough, haemoptysis, fever, hoarseness, night sweats and shortness of breath

In this case tumour metastasis was found in the heart. Although primary cardiac tumours are rare, metastasis in the heart is common. Due to its resemblance of the morphology and histology to the mediastinal tumour and the absence of possible explanation for a primary malignant melanoma of the heart, we suggest that the cardiac tumour is a secondary from a primary mediastinal tumour. Myocardial involvement by neoplasm arising elsewhere in the body is no longer considered rare [8]. Secondary or metastatic heart tumours occur comparatively more frequently, with at least a 100 times higher incidence than primary tumours [9].

Effects of metastases to the heart include a rapid increase in heart size by pericardial effusion, new signs of heart failure or valve disease, conduction defects, and atrial or ventricular heart rhythm disturbances [9]. Pericardial involvement of tumour cells causes pericarditis and serosanguinous or haemorrhagic pericardial effusion. Rapid filling of pericardial fluid leads to cardiac tamponade due to the low compliance of the pericardium to accommodate such an acute increase of pericardial fluid [9]. It causes severe haemodynamic compromise and death. It is treated with expansion of intravascular volume (small amounts of crystalloids or colloids may lead to 
improvement, especially in hypovolaemic patients) and urgent pericardial drainage [10].

Replacement of the myocardium by tumour cells and obliteration of the heart chambers by intra cavitatory growth of the tumour end up in cardiac failure. Invasion of the conducting tissues of the heart leads to atrial and ventricular rhythm abnormalities. Complete AV block causes syncope and sudden death. Tumour embolism to a coronary artery and invasion or compression of coronaries by tumour result in myocardial infarctions [9].

In this case the pericardial fluid at the time of death was $100 \mathrm{cc}$ and as little as $100 \mathrm{ml}$ of pericardial fluid can be fatal depending on the rapidity of its accumulation although 400$500 \mathrm{ml}$ of fluid is thought to be sufficient to cause fatal cardiac tamponade [11]. So the mechanism of death can be cardiac tamponade, arrhythmia, heart failure, myocardial infarction or sudden cardiac death.

\section{Conclusion}

We report this case as a primary malignant melanoma of the mediastinum with a right atrial metastasis causing pericardial effusion, as there was no evidence of a primary pigmented lesion on the skin or at any other site in spite of thorough pathological evaluation.

\section{References}

1. Strollo DC, Christenson RD, Jett JR. Primary mediastinal tumours. Part 1. Tumours of the anterior mediastinum. Chest 1997 Aug; 112(2): 511-522

2. Temes R, Chavez T, Mapel D, Ketal L, Crowell $R$, Key C, Follis F, Pett $S$, Warnly J. Primary mediastinal malignancies; Findings in 219 patients. West J med 1999 march; 170(3): 161-166

3. Kalra A, Kalra A, Palaniswami C, Gajendra E, Rajput V. Primary malignant melanoma presenting as superior mediastinal mass. Int J Surg Case Rep 2011; 2(8): 239-240

4. Adebonojo SA, Grillo IA, Durodola JI. Primary malignant melanoma of the bronchus. Journal of the national medical association 1979; 71(6): 579-581

5. Neri S, Komatsu T, Kitamura J, Otsuka K, Katakami N, Tkahashi Y. Malignant melanoma of the lung. Report of two cases. Ann Thoracic Cardiovascsurg 2011; 17(2): 170-171

6. Park SY, Kim MY, Chae EJ. Primary malignant melanoma of the mediastinum. KJR 2012; 13(6) : 823-826.

7. Farrel DJ, Kashyap AP, Ashcroft T, Morrit GN. Primary malignant melanoma of the bronchus. Thorax 1996; 51: 223-224

8. Young JM, Goldman JR. Tumour metastasis to heart. $J$ of the American heart Association 1954; 9: 220-227.

9. Reynen K, Kockeritz $U$, Strasser RH. Metastasis to the heart. Annals of Oncology 2004; 15: 375-381.

10. Hoit BD. Pericardial disease and pericardial tamponade. Crit Care Med. Aug 2007;35(8 Suppl):S355-64

11. Ebert LC, Ampanozi G, Ruder TD, Hatch G, Thali MJ, Germerott T. CT based volume measurement and estimation in case of pericardial effusion. $J$ of forensic and Legal Medicine 2012; 19: 126-131.

\section{Contribution of authors}

Performing the autopsy-LMGN, MHM

Opinion- LMGN, ML

Writing the manuscript -LMGN

Revising the manuscript- FLBM, ML 